\title{
Prevalence and Associated Risk Factors for Anaemia Among Pregnant Women Attending Ante- Natal Clinic, Eswatini
}

\author{
Rumbidzai Dodzo ( $\nabla$ dodzorc@gmail.com ) \\ University of KwaZulu-Natal \\ Ropo Ebenezer Ogunsakin \\ University of KwaZulu-Natal \\ Themba G. Ginindza \\ University of KwaZulu-Natal
}

\section{Research Article}

Keywords: Anaemia, pregnant women, prevalence, risk factors, Eswatini

Posted Date: September 13th, 2021

DOl: https://doi.org/10.21203/rs.3.rs-753198/v1

License: (c) (i) This work is licensed under a Creative Commons Attribution 4.0 International License.

Read Full License 


\section{Abstract}

Background: Anaemia is one of the most common nutritional deficiency diseases observed globally and it affects about a third of the world's population. Anaemia in pregnant women is a worldwide public health concern that has severe consequences for both mothers and infants, including maternal death and foetal and infant mortality. In Low-income countries (LICs), $25 \%$ of indirect maternal mortality and $30 \%$ of neonatal deaths are due to anaemia in pregnancy. Therefore, this study aimed to determine the prevalence of anaemia and assess associated factors among pregnant women attending ante-natal care (ANC) clinic in the Kingdom of Eswatini, which might help for screening, prevention and treatment of anaemia and ultimately prevent its adverse effects.

Method: A total of 550 pregnant women between 15 and 49 years from three health facilities were randomly enrolled using a cross-sectional study design, from January to March 2021. Non-probability sampling approach was used to select the appropriate study unit. A face-toface interview was done using a structured questionnaire to collect data. Multiple logistic regression was performed to assess the factors associated with anaemia among the women.

Results: The overall anaemia prevalence among pregnant women was $43.1 \%$ (95\% Cl: 38.947.3). The mild, moderate and severe cases of anaemia were $21.3 \%$ (95\%Cl: 17.9-24.9); $21.1 \%(95 \% \mathrm{Cl}$ : 17.8-24.7) and $0.7 \%(95 \% \mathrm{Cl}: 0.2-1.9)$, respectively. The prevalence of anaemia was high among women aged 20-24 (11.1\%, 95\%Cl: 8.6-14.0) and 25-29years (11.1\%, 95\%Cl: 8.6-14.0). Factors associated with anaemia included living in the urban area $(\mathrm{OR}=1.8,95 \% \mathrm{Cl}$ : 1.19-2.72, $\mathrm{p}=0.005)$ and having anaemia 6 months before pregnancy $(\mathrm{OR}=4.64,95 \% \mathrm{Cl}: 1.1518 .71, \mathrm{p}=0.031)$. Additionally, gestational age at first ANC also was positively associated with anaemia: $3^{\text {rd }}$ trimester $(\mathrm{OR}=10.42,95 \% \mathrm{Cl}: 4.27-25.4, p<0.001)$ and $2^{\text {nd }}$ trimester $(\mathrm{OR}=1.62,95 \% \mathrm{Cl}: 1.02-2.60, \mathrm{p}=0.043)$

Conclusion: Anaemia remains prevalent among pregnant women in Eswatini. Thus, a comprehensive anaemia prevention program would be justified and could lower the country's burden of anemia.

\section{Background}

Anaemia is a significant public health concern, affecting about two billion people worldwide, among which 56 million are pregnant women $(1,2)$. Globally, anaemia has been estimated to affect about 800 million children and women. Adolescent girls and women are at a higher risk due to menstruation and the high demand for metabolism during pregnancy (3). About $42 \%$ of pregnant women are affected by anaemia worldwide (4). In Southeast Asia, the prevalence of anaemia among pregnant women is $48 \%$, and anaemia in pregnancy causes half of the global maternal deaths. India contributes to $80 \%$ of these deaths $(5,6)$. South America has the lowest prevalence of $24.1 \%$ (6). In Africa, studies have found an anaemia prevalence of $57.1 \%$ among pregnant women (2). Previous studies conducted in Tanzania have shown a varying prevalence of anaemia among pregnant women, ranging from 18-68\% (7). The overall prevalence of anaemia among pregnant women was $41 \%$ in Ethiopia and $45 \%$ in Ghana $(8,9)$. Although 
there is limited data for Southern Africa, a study in South Africa found a prevalence of $42.7 \%(10)$. There is no record of previous studies on anaemia in pregnancy in Eswatini. According to the World Health Organisation (WHO), anaemia among pregnant women is a significant health problem in LICs (11). WHO has defined anaemia in pregnancy as haemoglobin $(\mathrm{Hb})$ levels of less than $11 \mathrm{~g} / \mathrm{dl}(5,12)$. The different $\mathrm{Hb}$ levels for each anaemia class during pregnancy are 10.0-10.9 g/ $\mathrm{dl}$ for mild, 7-9.9 g/dl for moderate, and less than $7 \mathrm{~g} / \mathrm{dl}$ for severe anaemia (13). Additionally, Sub-Saharan Africa (SSA) has an estimated prevalence of $56 \%$, compared to the $22 \%$ of high-income countries (HICs) $(14,15)$.

In LICs, anaemia in pregnancy significantly contributes to maternal and infant morbidity and mortality (16). Anaemia in pregnancy is considered a risk factor for the poor pregnancy outcome, resulting in lifethreatening complications for both mother and foetus $(12,14)$. Foetal consequences include stillbirths, low weight, intrauterine growth restriction, premature babies, perinatal mortality, and neonatal sepsis (17, 18). In late pregnancy, anaemia results in poor foetal iron stores, which can irreversibly affect the brain and neurotransmitters in the foetus and postnatal babies, leading to developmental disorders (19). Effects of anaemia during pregnancy may be related to its severity; for example, mild anaemia may not affect the current pregnancy but may reduce maternal iron stores and affect subsequent pregnancies (7). In addition, anaemic pregnant women are more prone to many complications, including decreased work productivity, increased risk of cardiac diseases, preterm labour and delivery, postpartum haemorrhage, impaired immune function, and maternal mortality $(7,11)$. Anaemia during pregnancy has been estimated to account for $23 \%$ of the indirect causes of maternal deaths in developing countries $(6,19)$.

Moreover, several factors have been identified as contributing factors of anaemia among pregnant women. Iron deficiency is the most common cause; it is usually accompanied by a deficiency of other nutrients $(8,13)$. Around $40 \%$ of women begin their pregnancy with decreased iron stores, which becomes insufficient to meet the increased iron needs during pregnancy $(2,20)$. Other factors include physiological haemodilution, underlying inflammatory conditions, and malnutrition, leading to insufficient vitamins, proteins, iron, and iodine $(11,13)$. In addition, in SSA, infections like malaria, helminths, and Human Immunodeficiency Virus (HIV) also contribute to anaemia in pregnancy $(13,21)$. Despite the broader scope of the problem, little research data has been explored about the severity of anaemia at ANC clinic in this study area; hence, there is a need to identify and treat anaemia to avoid its complications in pregnancy $(1,11)$. Furthermore, assessing the different factors contributing to anaemia in pregnancy is essential for effective anaemia management during pregnancy and provides the information necessary for planning and policy-making $(7,9)$. Despite its known effect on the population, the available data regarding the determinants of anaemia during pregnancy in the Kingdom of Eswatini is limited. Hence, this study aimed to determine the prevalence and risks associated with anaemia among pregnant women attending ante-natal clinics (ANC) in the three health facilities in the Kingdom of Eswatini to provide information on screening, prevention, and treatment of anaemia.

\section{Methods And Materials}

\section{Study design and population}


The study participants were pregnant women aged 15-49 years, attending ANC in healthcare facilities from January to March 2021. The women were recruited from three randomly selected healthcare facilities (Mankayane Government Hospital, Mbabane Government Hospital, and Raleigh Fitkin Memorial (RFM) Hospital) in the Kingdom of Eswatini. All pregnant women attending ANC in these three healthcare facilities, meeting the inclusion criteria and provided written informed consent were included.

\section{Sample size}

To estimate the prevalence of anaemia, assuming $95 \%$ confidence and an acceptable margin of error of $5 \%$ and maximum variability, i.e., $50 \%$ (given unknown prevalence), a sample size of 384 participants was required. The sample size was further increased by a margin of $10 \%$ to account for potential refusal and multiplied by a design effect (D) of 1.3, and the final sample size of the study was 550 pregnant women.

\section{Sampling strategy}

The recruitment of participants was done using non-probability sampling, where participants were recruited as they came in and as long as they met the inclusion criteria. Informed consent was obtained from the participants, and enrolment was equally offered to the participants irrespective of culture, religion, race, and social class. Trained research assistants explained the study aims and procedures to the women willing to participate while waiting to receive health services. As part of obtaining informed consent, potential risks or possible discomforts associated with participating in the study were discussed. After obtaining written informed consent, the recruitment of participants began, which were all pregnant women aged 15-49 years seeking ANC services from the study sites. Recruitment continued until the desired sample size was reached. All women who met the criteria were recruited into the study. Those who agreed to participate were assigned an anonymous participant identification number (e.g., C01-P001 for Mankayane Government Hospital; Patient number 1). There was no linkage with the patient's name and other personally identifiable information with the study findings and results, but only through the participant identification number.

\section{Data collection procedure}

A face-to-face interview using a structured questionnaire was used by research assistants to collect the relevant information in a consultation room where privacy was considered. The questionnaire included sections on socio-demographic characteristics, obstetrics information, nutritional data, and medical history. Data were entered and stored into SPSS version 27. In addition, each participant was assigned a unique identifier which was used to link the questionnaire and the results.

\section{Specimen collection and testing}

The specimen used for analysis was an ethylenediaminetetraacetic acid (EDTA) tube collected by venipuncture by the laboratory staff for full blood count (FBC). These specimens were processed in the laboratories of the same hospitals (Mankayane, Mbabane, and RFM). The Hb result (from the FBC) was used to determine whether the patient was anaemic or not, using the WHO criteria of $\mathrm{Hb}$ concentration < $11 \mathrm{~g} / \mathrm{dl}$ in pregnant women. The degree of severity of anaemia in pregnancy was classified into three: 
- Mild anaemia: $10.0-10.9 \mathrm{~g} / \mathrm{dl}$

- Moderate anaemia: $7.0-9.9 \mathrm{~g} / \mathrm{dl}$

- Severe anaemia: $<7.0 \mathrm{~g} / \mathrm{dl}$

\section{Quality control}

Questionnaires were pre-tested prior to the actual data collection. The collected data were checked for consistency and completeness daily. All procedures and methods were performed in accordance with the relevant guidelines, regulations and standard operating procedures (SOPs).

\section{Statistical analysis}

Data were processed and analysed using the STATA version15.0 (Stata Corp. College Station, Texas, USA). Data were checked for possible errors and any missing values before analysis. Descriptive and inferential statistics were used for analysis. Categorical variables were summarised using frequencies and proportions, while continuous variables were presented using mean and standard deviation. To identify factors associated with the outcome of interest, a bivariate logistic regression analysis was performed for each independent variable, and crude odds ratio (COR) with $95 \%$ confidence intervals was obtained. The strength of statistical association was measured by adjusted odds ratio (AOR) and $95 \%$ confidence intervals.

\section{Ethical consideration}

The study was approved by the Eswatini Health and Human Research Review Board

(EHHRRB) (SHR264/2020) and UKZN Biomedical Research Ethics Committee (BREC/00002158/2020). The ethics committees approved the form used to obtain written informed consent from the participants before participating in the study. The study purpose, objectives, and procedures were explained to the patients before signing the consent form. Therefore, all pregnant women aged $15-49$ years seeking ANC services from the study sites were eligible for the study. According to the Eswatini national guidelines for HIV testing and counselling, the consenting age is 12 years (22).

\section{Results}

\section{Characteristics of the study population}

A total of 550 pregnant women aged 15-49 years were enrolled in the study from January to March 2021 from three randomly selected hospitals (Mankayane Government, Mbabane Government, and RFM) in the Kingdom of Eswatini. The mean ( \pm standard deviation [SD]) age for the enrolled women was $27.2( \pm 6.4)$ years (Table 1 ). Above half (52.9\%) of the participants were aged $20-29$ years, $68.4 \%$ had high school level education, and $61.8 \%$ were unemployed. The majority $(65.1 \%)$ of the women were single, and $56.6 \%$ lived in urban areas. 
Approximately $80 \%$ of the participants' household monthly income was less than $\$ 343$, with many family members in the household mean of $4.0( \pm 2.3)$. The gestational age was from 5 to 40 weeks, with a mean $( \pm$ SD) of $29.0( \pm 7.74)$ weeks. About $36.6 \%$ of the participants were pregnant for the first time, and $40.4 \%$ reported an inter-pregnancy interval of $\geq 4$ years. The mean $( \pm S D)$ age at first pregnancy was $21.0( \pm$ 3.9 ), ranging from 14 to 39 years. In addition, $74.9 \%$ were taking iron supplements during their current pregnancy. $40.4 \%$ of participants ate meat, $33.8 \%$ dairy products, $85.5 \%$ fruits and vegetables $\geq$ four times during the week, and $51.8 \%$ were not eating fish. The women's mid-upper arm circumference ranged from 19 to $43 \mathrm{~cm}$, with a mean $( \pm S D)$ of $27.4( \pm 3.8)$. A large number of the participants were not infected with HIV (68.7\%), were not on any chronic medication (66.7\%), and did not have any sexually transmitted infections (98.4\%) or worm infestation (99.3\%), 6 months before the study. The haemoglobin results ranged from $5.9-15.5 \mathrm{~g} / \mathrm{dl}$ with a mean $( \pm \mathrm{SD})$ of $11.1( \pm 1.6)$. In addition, $96.7 \%$ of participants had no history of anaemia in previous pregnancies, while $97.5 \%$ did not have anaemia 6 months before the study (Table 1). 
Table 1

Socio-demographic characteristics of the study population $(N=550)$

\begin{tabular}{|c|c|}
\hline Variable & n (\%) \\
\hline \multicolumn{2}{|l|}{ Age: n (\%) } \\
\hline $\operatorname{Mean}( \pm \mathrm{SD})$ & $27.2( \pm 6.4)$ \\
\hline $15-19$ & $75(13.6)$ \\
\hline $20-29$ & $291(52.9)$ \\
\hline $30-39$ & $173(31.5)$ \\
\hline $40-49$ & $11(2.0)$ \\
\hline Family members in household: Mean( \pm SD) & $4.0( \pm 2.3)$ \\
\hline \multicolumn{2}{|l|}{ Residence: n(\%) } \\
\hline Rural & $239(43.5)$ \\
\hline Urban & $311(56.6)$ \\
\hline \multicolumn{2}{|l|}{ Marital status: $n(\%)$} \\
\hline Single & $358(65.1)$ \\
\hline Married & $192(34.9)$ \\
\hline \multicolumn{2}{|l|}{ Employment status: $n(\%)$} \\
\hline Unemployed & $340(61.8)$ \\
\hline Employed & $173(31.5)$ \\
\hline Self-employed & $37(6.7)$ \\
\hline \multicolumn{2}{|l|}{ Level of education: $n(\%)$} \\
\hline Never been to school & $5(0.9)$ \\
\hline Primary & $85(15.5)$ \\
\hline High school & $376(68.4)$ \\
\hline Tertiary & $84(15.3)$ \\
\hline \multicolumn{2}{|l|}{ Household monthly income: $n(\%)$} \\
\hline Less than E5000 (\$343) & $434(78.9)$ \\
\hline E5000-E10 000 & $84(15.3)$ \\
\hline More than E10 000 & $32(5.8)$ \\
\hline
\end{tabular}




\begin{tabular}{|c|c|}
\hline Variable & n (\%) \\
\hline $\operatorname{Mean}( \pm$ SD) & $28.0( \pm 7.7)$ \\
\hline 1-12 (1 st trimester) & $18(3.3)$ \\
\hline 13-26 (2nd trimester) & $201(36.6)$ \\
\hline 27-40 (3rd trimester) & $331(60.2)$ \\
\hline Gestational age at 1 st ANC: Mean $( \pm$ SD) & $17.4( \pm 6.2)$ \\
\hline Age at 1st pregnancy: Mean ( \pm SD) & $21.0( \pm 3.9)$ \\
\hline \multicolumn{2}{|l|}{ Gravidity: n(\%) } \\
\hline 1 & 201 (36.6) \\
\hline 2 & 145 (26.4) \\
\hline 3 & $99(18.0)$ \\
\hline 4 and above & 105 (19.1) \\
\hline \multicolumn{2}{|l|}{ Parity: n(\%) } \\
\hline 0 & $214(38.9)$ \\
\hline 1 & $151(27.5)$ \\
\hline 2 & $98(17.8)$ \\
\hline 3 & $58(10.5)$ \\
\hline 4 and above & $29(5.3)$ \\
\hline \multicolumn{2}{|l|}{ Number of ANC visits: $n(\%)$} \\
\hline 1 & $122(22.2)$ \\
\hline 2 & $131(23.8)$ \\
\hline 3 & $134(24.4)$ \\
\hline 4 and above & $163(29.6)$ \\
\hline \multicolumn{2}{|l|}{ Inter-pregnancy interval: n(\%) } \\
\hline No previous child & $214(38.9)$ \\
\hline 1 & $23(4.2)$ \\
\hline 2 & $35(6.4)$ \\
\hline 3 & $56(10.2)$ \\
\hline 4 and above & $222(40.4)$ \\
\hline
\end{tabular}




\begin{tabular}{|c|c|}
\hline Variable & n (\%) \\
\hline \multicolumn{2}{|l|}{ Taking Iron supplements: $\mathrm{n}(\%)$} \\
\hline Yes & $412(74.9)$ \\
\hline No & $138(25.1)$ \\
\hline Mid-upper arm circumference (MUAC): Mean ( \pm SD) & $27.4( \pm 3.8)$ \\
\hline \multicolumn{2}{|l|}{ Meat consumption per week: $\mathrm{n}(\%)$} \\
\hline Does not eat & $12(2.2)$ \\
\hline 1 & $76(13.8)$ \\
\hline 2 & $92(16.7)$ \\
\hline 3 & $148(26.9)$ \\
\hline 4 and above & $222(40.4)$ \\
\hline \multicolumn{2}{|l|}{ Fish consumption per week: $\mathrm{n}(\%)$} \\
\hline Does not eat & $285(51.8)$ \\
\hline 1 & $177(32.2)$ \\
\hline 2 & $54(9.8)$ \\
\hline 3 & $19(3.5)$ \\
\hline 4 and above & $15(2.7)$ \\
\hline \multicolumn{2}{|l|}{ Fruit and vegetables consumption per week: $n(\%)$} \\
\hline 1 & $8(1.5)$ \\
\hline 2 & $23(4.2)$ \\
\hline 3 & 49 (8.9) \\
\hline 4 and above & $470(85.5)$ \\
\hline \multicolumn{2}{|l|}{ Dairy consumption per week: $\mathrm{n}(\%)$} \\
\hline Does not eat & $36(6.6)$ \\
\hline 1 & $129(23.5)$ \\
\hline 2 & 104 (18.9) \\
\hline 3 & 95 (17.3) \\
\hline 4 and above & $186(33.8)$ \\
\hline Haemoglobin $(\mathrm{Hb})$ : Mean $( \pm \mathrm{SD})$ & $11.1( \pm 1.59)$ \\
\hline
\end{tabular}




\begin{tabular}{|ll|}
\hline Variable & $\mathbf{n}(\%)$ \\
\hline HIV status: $\mathbf{n}(\%)$ & $378(68.7)$ \\
\hline Negative & $172(31.3)$ \\
\hline Positive & \\
\hline STI in the last 6 months: $\mathbf{n}(\%)$ & $541(98.4)$ \\
\hline No & $9(1.6)$ \\
\hline Yes & \\
\hline Worm infestation in the last 6 months: $\mathbf{n}(\%)$ & $546(99.3)$ \\
\hline No & $4(0.7)$ \\
\hline Yes & $367(66.7)$ \\
\hline Are you on chronic medication: $\mathbf{n}(\%)$ & $183(33.3)$ \\
\hline No & \\
\hline Yes & $532(96.7)$ \\
\hline Anaemia in previous pregnancy: $\mathbf{n}(\%)$ & $18(3.3)$ \\
\hline No & \\
\hline Yes & \\
\hline Anaemia 6 months before pregnancy: $\mathbf{n}(\%)$ & $(2.6)$ \\
\hline No & \\
\hline Yes & \\
\hline
\end{tabular}

\section{Anaemia prevalence}

The overall anaemia prevalence was $43.1 \%$ (95\% Cl: 38.9-47.3). The mild, moderate and severe cases of anaemia were $21.3 \%$ (95\% Cl: $17.9-24.9) ; 21.1 \%$ (95\% Cl: $17.8-24.7)$ and $0.7 \%$ (95\% Cl: $0.2-1.9)$, respectively. Anaemia prevalence was higher among women living in the urban area $(27.6 \%, 95 \% \mathrm{Cl}$ : $23.9-$ 31.6) as compared to women living in rural area $(15.5 \%, 95 \% \mathrm{Cl}: 12.5-18.8)$ (Table 2$)$. The prevalence of

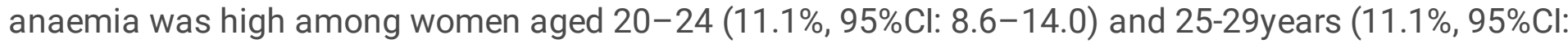
8.6-14.0). Single women had a higher prevalence, $31.1 \%(95 \% \mathrm{Cl}: 27.2-35.1)$ as compared to those who were married, $12.0 \%$ (95\% Cl: $9.4-15.0)$. The highest prevalence was among the unemployed $(27.5 \%$, $95 \% \mathrm{Cl}$ : 23.831.4), those who had a high school education $(29.8 \%, 95 \% \mathrm{Cl}: 26.0-33.8)$ and women who had a household monthly income of less than E5000 (\$343) (35.5\%, 95\%Cl: 31.5-39.6). The highest prevalence was among those who were pregnant for the first time $(17.8 \%, 95 \% \mathrm{Cl}$ : $14.7-$ 
21.3). The prevalence declined with increasing gravidity (gravida 1 (16.7\%, 95\% Cl: 13.720 .1$) ; 2(11.1 \%$, $95 \% \mathrm{Cl}: 8.6-14.0)$ and $3(7.8 \%, 95 \% \mathrm{Cl}: 5.7-10.4)$; increasing parity (para $0(17.8 \%, 95 \% \mathrm{Cl}: 14.7-21.3) ; 1$ (11.6\%, 95\%Cl: $9.1-14.6)$ and $2(6.9 \%, 95 \% \mathrm{Cl}: 4.9-9.4)$ and age at 1 st pregnancy $(15-19(18.7 \%, 95 \% \mathrm{Cl}$ : 15.6-22.2); 25-29 (5.3\%, 95\% Cl: 3.6-7.5) and 30-34 (0.9\%, 95\% Cl: 0.3-2.1). It increased with an increase in gestational age (1st trimester (1.1\%, 95\% Cl: $0.4-2.4)$; 2 nd trimester (14.0\%, 95\% Cl: $11.2-17.2)$ and 3rd trimester $(28.0 \%, 95 \% \mathrm{Cl}: 24.3-32.0)$. At first ANC visit, the prevalence was highest in the second trimester (29.5\%, 95\% Cl: 25.7-33.5) (Table 2).

Table 2: The prevalence of anaemia among pregnant women (15-49 years) in Eswatini ( $N=550)$ 
Variable

Overall prevalence

Age (years)

15-19

20-24

25-29

30-34

35-39

40-44

Family members in household

1-4

5-9

10-14

\section{Residence}

Rural

Urban

\section{Marital status}

Single

Married

\section{Employment status}

Unemployed

Employed

Self-employed

\section{Level of education}

Never been to school

Primary

High school

Tertiary

Household monthly income
Anaemic $(\mathrm{N}=550) \quad \%(95 \% \mathrm{Cl})$

P-value

237

43.1\% (95\% Cl: 38.9-47.3)

$7.3(5.2-9.8)$

$11.1(8.6-14.0)$

11.1 (8.6-14.0)

0.039

$6.4(4.5-8.7)$

$6.9(4.9-9.4)$

$0.4(0.04-1.3)$
29.1 (25.3-33.1)

$13.6(10.9-16.8)$

0.025

$0.4(0.04-1.3)$

$15.5(12.5-18.8)$

$27.6(23.9-31.6)$

0.002

152

$31.1(27.2-35.1)$

$12.0(9.4-15.0)$

0.003

66

151

$27.5(23.8-31.4)$

72

$13.1(10.4-16.2)$

0.667

14

$2.5(1.4-4.2)$

$0.4(0.04-1.3)$

7.5 (5.4-10.0)

29.8 (26.0-33.8)

0.416
164

30

5.5 (3.7-7.7) 
Less than E5000 (\$343)

E5000-E10 000

More than E10 000

Gestational age

$1-12$ ( $1^{\text {st }}$ trimester)

$13-26$ ( $2^{\text {nd }}$ trimester)

$27-40$ ( $3^{\text {rd }}$ trimester)

Gestational age at $1^{\text {st }}$ ANC

$1-12\left(1^{\text {st }}\right.$ trimester $)$

$13-26\left(2^{\text {nd }}\right.$ trimester)

$27-40$ ( $3^{\text {rd }}$ trimester $)$

Age at $1^{\text {st }}$ pregnancy

15-19

103

97

29

5

30-34

35-39

40-44

Gravidity

195

31

11

6

77

154

37

162

38

1

2
$6.7(4.8-9.2)$

$29.5(25.7-33.5)$

$<0.001$

$6.9(4.9-9.4)$

$18.7(15.6-22.2)$

$17.6(14.5-21.1)$

$5.3(3.6-7.5)$

0.613

$0.9(0.3-2.1)$

$0.2(0.0-1.0)$

0.4 (0.04-1.3)
0.234

0.125

$28.0(24.3-32.0)$

$\begin{array}{ll}1 & 0.2(0.0-1.0) \\ 2 & 0.4(0.04-1.3)\end{array}$




\begin{tabular}{llll}
1 & 92 & $16.7(13.7-20.1)$ & \\
\hline 2 & 61 & $11.1(8.6-14.0)$ & 0.716 \\
\hline 3 & 43 & $7.8(5.7-10.4)$ & \\
\hline 4 and above & 41 & $7.5(5.4-10.0)$ &
\end{tabular}

\section{Parity}

\begin{tabular}{llll}
0 & 98 & $17.8(14.7-21.3)$ & \\
\hline 1 & 64 & $11.6(9.1-14.6)$ & \\
\hline 2 & 38 & $6.9(4.9-9.4)$ & 0.632 \\
\hline 3 & 27 & $4.9(3.3-7.1)$ & \\
\hline 4 and above & 10 & $1.8(0.9-3.3)$ &
\end{tabular}

\section{Number of ANC visits}

\begin{tabular}{llll}
\hline 1 & 53 & $9.6(7.3-12.4)$ & \\
\hline 2 & 58 & $10.5(8.1-13.4)$ & 0.525 \\
\hline 3 & 63 & $11.5(8.9-14.4)$ & \\
\hline 4 and above & 63 & $11.5(8.9-14.4)$ & \\
\hline \multicolumn{1}{l}{ Inter-pregnancy interval } & & & \\
\hline No previous child & 98 & $17.8(14.7-21.3)$ & \\
\hline 1 & 15 & $2.7(1.5-4.5)$ & \\
\hline 2 & 12 & $2.2(1.1-3.9)$ & 0.115 \\
\hline 3 & 24 & $4.4(2.8-6.4)$ & \\
\hline 4 and above & 88 & $16(13.0-19.3)$ & \\
\hline
\end{tabular}

\section{Taking Iron supplements}

\begin{tabular}{llll} 
Yes & 175 & $31.8(27.9-35.9)$ & 0.615 \\
No & 62 & $11.3(8.6-14.2)$ & \\
\hline
\end{tabular}

\section{Fish consumption per week}

\begin{tabular}{llll} 
Does not eat & 130 & $23.6(20.1-27.4)$ & \\
\hline 1 & 78 & $14.2(11.4-17.4)$ & \\
\hline 2 & 18 & $3.3(2.0-5.1)$ & 0.341 \\
\hline 3 & 6 & $1.1(0.4-2.4)$ & \\
& & &
\end{tabular}




\section{Fruit\& vegetables consumption per week}

\begin{tabular}{llll}
\hline 1 & 4 & $0.7(0.2-1.9)$ & \\
\hline 2 & 7 & $1.3(0.5-2.6)$ & 0.628 \\
\hline 3 & 22 & $4.0(2.5-6.0)$ & \\
\hline 4 and above & 204 & $37.1(33.0-41.3)$ & \\
\hline
\end{tabular}

\section{Dairy consumption per week}

$\begin{array}{llll}\text { Does not eat } & 15 & 2.7(1.5-4.5) & \\ 1 & 57 & 10.4(7.9-13.2) & \\ 2 & 56 & 10.2(7.8-13.0) & 0.115 \\ 3 & 39 & 7.1(5.1-9.6) & \\ 4 \text { and above } & 70 & 12.7(10.1-15.8) & \end{array}$

\section{HIV status}

$\begin{array}{llll}\text { Positive } & 100 & 18.2(15.0-21.7) & <0.001 \\ \text { Negative } & 137 & 24.9(21.3-28.7) & \end{array}$

\section{STI in the last 6 months}

$\begin{array}{llll}\text { Yes } & 7 & 1.3(0.5-2.6) & 0.034 \\ \text { No } & 230 & 41.8(37.7-46.1) & \end{array}$

Worm infestation in the last 6 months

\begin{tabular}{lllll}
\hline Yes & 1 & $0.2(0.0-1.0)$ & 0.463 \\
\hline No & 236 & $42.9(38.7-47.2)$ & \\
\hline \multicolumn{1}{l}{ Are you on chronic medication } & & & \\
\hline Yes & 104 & $18.9(15.7-22.4)$ & $<0.001$ \\
\hline No & 133 & $24.2(20.7-28.0)$ & \\
\hline $\begin{array}{l}\text { Anaemia } \quad \text { in } \quad \text { previous } \\
\text { pregnancy }\end{array}$ & & & \\
\hline Yes & & & & \\
\hline No & & 11 & $2.0(1.0-3.6)$ & 0.116 \\
\hline
\end{tabular}

Anaemia 6 months before pregnancy 


\section{Risk factors associated with anaemia among pregnant women}

Table 3 shows the risk factors associated with anaemia among pregnant women. Based on the univariate analysis: increasing age (25-29yrs: $O R=0.52,95 \% \mathrm{Cl}: 0.30-0.90, p=0.02 ; 40-44 y r s \mathrm{OR}=0.19$, $95 \% \mathrm{Cl}: 0.04-0.96, p=0.045)$, being married $(\mathrm{OR}=0.57,95 \% \mathrm{Cl}: 0.4-0.82, \mathrm{p}=0.03)$ and increasing number of family members in household $(\mathrm{OR}=0.15,95 \% \mathrm{Cl}$ : $0.033-0.64, p=0.011)$ were inversely associated with anaemia. Living in the urban area $(\mathrm{OR}=1.73,95 \% \mathrm{Cl}$ : $1.23-2.45, \mathrm{p}=0.002)$, being on chronic medication $(\mathrm{OR}=2.32,95 \% \mathrm{Cl}: 1.61-3.33, \mathrm{p}<0.001)$, having anaemia 6 months before pregnancy $(\mathrm{OR}=5.03,95 \% \mathrm{Cl}$ : 1.39-18.24, $p=0.014)$ and being HIV positive $(\mathrm{OR}=2.44,95 \% \mathrm{Cl}: 1.69-3.53, \mathrm{p}<0.001)$ were positively associated with anaemia. Gestational age at first ANC visit was also positively associated with anaemia where those in the 3rd trimester ( $O R=9.59,95 \% \mathrm{Cl}: 4.21-21.83, p<0.001)$ were about 6 -fold more likely to be anaemic as compared to those in the 2 nd trimester $(\mathrm{OR}=1.67,95 \% \mathrm{Cl}: 1.08-2.59, \mathrm{p}=0.021)$.

Additionally, going through the result of multivariate analysis, increasing age $(25-29 \mathrm{yrs}$ : $\mathrm{OR}=0.43,95 \% \mathrm{Cl}$ : $0.23-0.81, p=0.008 ; 40-44 y r s$ OR $=0.15,95 \% \mathrm{Cl}: 0.02-0.85, p=0.033)$, being married $(\mathrm{OR}=0.61,95 \% \mathrm{Cl}$ : $0.38-0.98, p=0.039)$ and increasing number of family members in household $(\mathrm{OR}=0.18,95 \% \mathrm{Cl}$ : $0.036-$ $0.92, p=0.039)$ remained inversely associated with anaemia. Living in the urban area and having anaemia 6 months before pregnancy $(\mathrm{OR}=1.8,95 \% \mathrm{Cl}: 1.19-2.72, \mathrm{p}=0.005$ and $\mathrm{OR}=4.64,95 \% \mathrm{Cl}: 1.15-$ $18.71, p=0.031$, respectively) remained statistically significant factors of anaemia. Gestational age at first ANC visit also remained positively associated with anaemia where those in the 3rd trimester (OR = $10.42,95 \% \mathrm{Cl}: 4.27-25.4, \mathrm{p}<0.001)$ were about 6 -fold more likely to be anaemic as compared to those in the 2 nd trimester $(\mathrm{OR}=1.62,95 \% \mathrm{Cl}: 1.02-2.60, \mathrm{p}=0.043)$ (Table 3$)$. 
Table 3

Risk factors associated with anaemia among pregnant women (15-49 years) in Eswatini ( $N=550)$

\begin{tabular}{|c|c|c|c|c|c|}
\hline \multirow[t]{2}{*}{ Risk factors } & \multirow{2}{*}{$\begin{array}{l}\text { Anaemic } \\
\mathrm{n}(\%)\end{array}$} & \multicolumn{2}{|c|}{ Unadjusted (Univariate) } & \multicolumn{2}{|c|}{ Adjusted (Multivariable) } \\
\hline & & OR $(95 \% \mathrm{Cl})$ & Pvalue & OR $(95 \% \mathrm{Cl})$ & P-value \\
\hline Residence Rural & $85(15.5)$ & 1 (ref) & & 1 (ref) & \\
\hline Urban & $12(27.6)$ & $1.73(1.23-2.45)$ & 0.002 & $1.8(1.19-2.72)$ & 0.005 \\
\hline \multicolumn{6}{|l|}{ Marital Status } \\
\hline Single & $\begin{array}{l}171 \\
(31.1)\end{array}$ & 1 (ref) & & 1 (ref) & \\
\hline Married & $66(12)$ & $0.57(0.40-0.82)$ & 0.003 & $0.61(0.38-0.98)$ & 0.039 \\
\hline \multicolumn{6}{|l|}{ Age (years) } \\
\hline $15-19$ & $40(7.3)$ & 1 (ref) & & 1 (ref) & \\
\hline $20-24$ & $61(11.1)$ & $0.81(0.46-1.43)$ & 0.467 & $0.69(0.37-1.28)$ & 0.238 \\
\hline $25-29$ & $61(11.1)$ & $0.52(0.30-0.90)$ & 0.020 & $0.43(0.23-0.81)$ & 0.008 \\
\hline $30-34$ & $35(6.4)$ & $0.52(0.28-0.96)$ & 0.037 & $0.41(0.19-0.87)$ & 0.020 \\
\hline $35-39$ & $38(6.9)$ & $0.81(0.43-1.53)$ & 0.516 & $0.60(0.28-1.31)$ & 0.204 \\
\hline $40-44$ & $2(0.4)$ & $0.19(0.04-0.96)$ & 0.045 & $0.15(0.02-0.85)$ & 0.033 \\
\hline \multicolumn{6}{|l|}{$\begin{array}{l}\text { STI in the last } 6 \\
\text { months }\end{array}$} \\
\hline No & $\begin{array}{l}230 \\
(41.8)\end{array}$ & 1 (ref) & & 1 (ref) & \\
\hline Yes & $7(1.3)$ & $\begin{array}{l}4.73(0.97- \\
22.99)\end{array}$ & 0.054 & $3.9(0.63-24.06)$ & 0.143 \\
\hline \multicolumn{6}{|c|}{ Are you on chronic medication } \\
\hline No 133 (24.2) & & 1 (ref) & & 1 (ref) & \\
\hline Yes 104 (18.9) & & $2.32(1.61-3.33)$ & $<0.001$ & $1.37(0.36-5.21)$ & 0.641 \\
\hline \multicolumn{6}{|c|}{ Anaemia 6 months before pregnancy } \\
\hline No 226 (41.1) & & 1 (ref) & & 1 (ref) & \\
\hline Yes $11(2.0)$ & & $\begin{array}{l}5.03(1.39- \\
18.24)\end{array}$ & 0.014 & $4.64(1.15-18.71)$ & 0.031 \\
\hline \multicolumn{6}{|c|}{ Family members in household } \\
\hline $1-4$ & $\begin{array}{l}160 \\
(29.1)\end{array}$ & 1 (ref) & & 1 (ref) & \\
\hline
\end{tabular}




\begin{tabular}{|c|c|c|c|c|c|}
\hline \multirow{2}{*}{$\begin{array}{l}\text { Risk factors } \\
5-9\end{array}$} & \multirow{2}{*}{$\begin{array}{l}\text { Anaemic } \\
75(13.6)\end{array}$} & \multicolumn{2}{|c|}{ Unadjusted (Univariate) } & \multicolumn{2}{|c|}{ Adjusted (Multivariable) } \\
\hline & & $0.97(0.67-1.40)$ & 0.878 & $1.18(0.76-1.81)$ & 0.465 \\
\hline $10-14$ & $2(0.4)$ & $\begin{array}{l}0.15(0.033- \\
0.64)\end{array}$ & 0.011 & $0.18(0.036-0.92)$ & 0.039 \\
\hline \multicolumn{6}{|l|}{ HIV } \\
\hline Negative & $\begin{array}{l}137 \\
(24.9)\end{array}$ & 1 (ref) & & 1 (ref) & \\
\hline Positive & $\begin{array}{l}100 \\
(18.2)\end{array}$ & $2.44(1.69-3.53)$ & $<0.001$ & $2.17(0.56-8.44)$ & 0.263 \\
\hline \multicolumn{6}{|c|}{ Gestational age at 1st ANC } \\
\hline 1-12(1st trimester) & $37(6.7)$ & 1 (ref) & & 1 (ref) & \\
\hline 13-26(2nd trimester) & $\begin{array}{l}162 \\
(29.5)\end{array}$ & $1.67(1.08-2.59)$ & 0.021 & $1.62(1.02-2.60)$ & 0.043 \\
\hline $27-40$ (3rd trimester) & $38(6.9)$ & $\begin{array}{l}9.59(4.21- \\
21.83)\end{array}$ & $<0.001$ & $10.42(4.27-25.4)$ & $<0.001$ \\
\hline
\end{tabular}

\section{Discussion}

This is the first study assessing prevalence and risk factors for anaemia in pregnancy in the Kingdom of Eswatini. The present study shows a high prevalence of anaemia in pregnancy, 43.1\%. Differences in severity of anaemia was observed with mild, moderate, and severe cases of anaemia at $21.3 \%, 21.1 \%$, and $0.7 \%$, respectively. The prevalence of anaemia was high among women aged $20-24(11.1 \%)$ and $25-$ 29 years $(11.1 \%)$. The highest prevalence was among pregnant women for the first time (17.8\%), and it increased with an increase in gestational age. The prevalence declined with increasing gravidity, increasing parity, and age at 1 st pregnancy shows the risk factors associated with anaemia among pregnant women. The univariate analysis showed that increasing age, being married, and increasing family members in household were inversely associated with anaemia while living in the urban area, being on chronic medication, having anaemia 6 months before pregnancy, and being HIV positive were positively associated with anaemia. Gestational age at first ANC visit was also positively associated with anaemia where those in the $(O R=9.59)$ were about 6 -fold more likely to be anaemic than those in the 2nd trimester $(O R=1.67)$. Multivariate analysis showed that being in the 3rd trimester at first ANC visit was the most severe risk for anaemia in pregnancy $(O R=10.4)$.

According to WHO, the prevalence in this study (43.1\%) shows that this is a public health concern, which states that anaemia is a public health problem if its prevalence is $\geq 5.0 \%$, and a severe problem if prevalence is $\geq 40 \%(7,15)$. Other researchers have reported the prevalence of anaemia during pregnancy, ranging from $32-62.2 \%$ and $57 \%$ in Sub-Saharan Africa countries (15). Similar prevalence was found in South East Asian countries (48\%) (6), Northern Tanzania (47\%) (15), Kenya (40\%) (23), and South Africa 
(43\%) (4). Lower prevalence has been reported in Lesotho (33.2\%) (24), Botswana (34\%) (25), Ethiopia (36.6\%), and Nigeria (37.6\%) (17). Higher prevalence was reported in Eastern Kenya (57\%) (26), Pakistan (56.4\%) (27), and North East India (60\%) (6). The variations in prevalence may be due to different causes of anaemia, dietary differences, population differences, study design, and differences in methodology used in determining haemoglobin levels $(23,27)$. Regarding the differences in severity, the similar prevalence was reported in Northern Ghana (8) and Egypt (28), where the mild and moderate anaemia cases were similar.

In this study, anaemia prevalence was highest and the same among women aged 20-24 and 2529 years (11.1\%). These findings are consistent with a study in India reporting the highest prevalence of anaemia (63.3\%) in a similar age group range (6). Another study in Northern Ghana reported similar findings, where anaemia prevalence in the age group 20 years and below was $51.5 \%$ compared to $47.8 \%$ in those older than 35years (29). High prevalence in younger ages might have been due to the lack of awareness, poor knowledge of antenatal services, and failure to seek prenatal care early and take care of themselves during pregnancy $(5,17)$. However, there were contrasting reports from other African studies from Ethiopia and Nigeria, where the highest prevalence was reported among participants of the age group 30-39 years in Ethiopia and the Nigerian study reported the highest prevalence among participants in the age group of 20years and below $(9,17)$. This might have been because of increased body weakness, multiple pregnancies, labour, and being subjected to other illnesses leading to a predisposition to anaemia as age advances $(9,26)$. This study reported anaemia prevalence higher among women living in the urban area $(27.6 \%)$ than women living in rural areas (15.5\%). Consistent findings were reported in a study in Southern Nigeria (17). This is contrary to other studies in India and Northern Ethiopia, where anaemia prevalence was high among pregnant women living in rural areas compared to those living in urban areas $(6,30)$. This may be because of limited access to health facilities and limited resources for adequate and proper nutrition during pregnancy $(16,30,31)$. Based on the finding of this study, anaemia prevalence was highest in women with a monthly household income of less than $\$ 343$ (35.5\%). These findings are consistent with a study in Ethiopia, where women from lower socio-economic classes had a higher prevalence of anaemia than those from higher socio-economic classes $(2,18)$. The women from lower socio-economic status were perceived as unable to afford good quality food $(2,12)$. The highest prevalence was among the unemployed, pregnant women (27.5\%). A study in Nigeria also reported consistent findings, and they stated that the participants had little or no income to buy the right food required to prevent anaemia (17). One study in Kenya showed contrasting results of the high prevalence among the employed participants (26). They stated that the employed participants had no time to rest or attend ANC clinics compared to housewives (26). The highest anaemia prevalence was reported among pregnant who was in the 3rd trimester of their pregnancy. Consistent results were reported in Egypt (28) and Ghana (8). This might have been due to reducing iron stores due to increasing demand for iron for both the mother and foetus, as pregnancy progress $(2,18)$.

This study showed that being on chronic medication was a risk for developing anaemia in pregnancy. Similarly, a study from India noted an association between anaemic pregnant women with a chronic illness in pregnancy or the recent past (11). There is a relative deficiency of iron in the body; therefore, the 
body cannot effectively use iron to generate new blood cells. Iron reserves are already low in these patients $(11,32)$. Also, this study found that HIV was associated with anaemia in pregnancy, where those infected with HIV had a 2.5 chance of developing anaemia. In Uganda, a study showed similar findings, where pregnant women infected with HIV were twice more likely to have anaemia than their HIV-negative counterparts (33). HIV infection is associated with lower serum folic acid and ferritin levels, and the use of antiretroviral drugs, especially Zidovudine, is associated with anaemia $(4,33)$. There was a significant association between gestational age at first ANC and anaemia in pregnancy. In this study, those in the 3rd trimester was about 6 -fold more likely to be anaemic than those in the 2 nd trimester. Consistent findings were reported in Ethiopia, where women in the second and third trimesters were 3.1 and 3.7 times more likely to develop anaemia than those in the first trimester (2). During pregnancy, physiological haemodilution occurs as the pregnancy progresses, worsening anaemia in the third trimester (16). Furthermore, areas of residence, particularly those residing in urban area may also impact pregnancy women. For instance, pregnant women living in a rural area versus living in an urban area has been investigated as a potential contributing factor for risk of developing anaemia among women (31). In addition, a study from Ethiopia affirmed that pregnant women in rural areas were almost 2-times more at risk of developing anaemia than those in urban areas (30). The possible explanation for this could be attributed to a lack of proper information about ANC as well as inaccessibility to health care facilities (30).

\section{Strengths and limitations of the study}

The limitations of this study were that this was a hospital-based study, so results could only be generalized to women attending ANC and not all pregnant women in the population. In addition, the study design was cross-sectional, so it was impossible to identify and establish cause and effect relationships. Nevertheless, the findings shed some light on anaemia in pregnancy and the associated risks, and they provide a platform for further studies. The study's strengths were that the diagnosis of anaemia was based on laboratory analysis and did not depend on clinical assessment. This is the first study on prevalence and risks associated with anaemia in pregnancy, and it gives perspective to the burden of anaemia in the Kingdom. It also serves as a benchmark for further research into the role of other factors that may contribute to understanding anaemia in pregnancy.

\section{Conclusion}

The results of this study highlight prevalence and associated risk factors for anaemia among pregnant women attending ANC in Eswatini and indicate potential factors for interference to alleviate the anaemia burden in Eswatini. The findings show that anaemia among pregnant women attending ANC in Eswatini is associated with the place of living and gestational age at first ANC, among others. Also, this study concludes that the prevalence of anaemia is high among pregnant women in Eswatini. Furthermore, the study has provided essential information about anaemia and associated risk factors that can contribute to policy development and prevention strategies. Finally, the findings provide epidemiological knowledge about the distribution of anaemia. This is crucial to guide the continuous awareness and health 
education on the implication of anaemia and the importance of prevention and reducing the risk of anaemia in pregnancy.

\section{Abbreviations}

\begin{tabular}{|ll|}
\hline ANC & Ante-natal care \\
AOR & Adjusted Odds Ratio \\
\hline BREC & Biomedical Research Ethics Committee \\
CI & Confidence interval \\
COR & Crude Odds Ratio \\
\hline FBC & Full Blood Count \\
\hline EHHRRB & Eswatini Health and Human Research Review Board \\
Hb & Haemoglobin \\
HICs & High Income Countries \\
\hline HIV & Human Immunodeficiency Virus \\
\hline LICs & Low Income Countries \\
\hline OR & Odds ratio \\
RFM & Raleigh Fitkin Memorial \\
\hline SD & Standard deviation \\
\hline SSA & Sub-Saharan Africa \\
STI & Sexually transmitted infections \\
\hline UKZN University of KwaZulu-Natal \\
\hline WHO World Health Organisation \\
\hline
\end{tabular}

\section{Declarations}

\section{Acknowledgements}

The authors would like to thank the University of KwaZulu-Natal, Discipline of Public Health Medicine for the provision of resources towards this study. We would like to thank the Kingdom of Eswatini Ministry of Health for allowing us to implement the study and the pregnant mothers who took part in this study. We also thank the administrations and the Maternal and Child Health units of the three hospitals for allowing us to implement the study. 


\section{Authors' contributions}

RD conceived the study and design. RD and TGG simplified acquisition of the data. RD, and REO analysed the data. RD, TGG, and REO interpreted the data. RD, TGG, and REO drafted the manuscript. RD, TGG, and REO provided critical review and final approval of the manuscript. All authors read and approved the final manuscript.

\section{Funding}

This research did not receive any specific grant from funding agencies in the public, or not-for profit sectors. The study was self-funded.

\section{Availability of data and materials}

Data from this study is the property of the Government of Eswatini and University of KwaZuluNatal and cannot be made publicly available. All interested readers can access the data set from Eswatini Health and Human Research Review Board (EHHRRB) and the University of KwaZulu-Natal Biomedical Research Ethics Committee (BREC) from the following contacts:

The Chairperson, Ministry of Health (EHHRRB), P. O BOX 5, Mbabane, Eswatini, Tel: (+268) 2404 231, Fax: (+268) 24042092 Email: babazileshongwe@gmail.com or kaluamasi@gmail.com. The Chairperson, Biomedical Research Ethics Administration, Research Office, Westville Campus, Govan Mbeki Building, University of KwaZulu-Natal

P/Bag X54001, Durban, 4000 KwaZulu-Natal, South Africa, Tel.: +27 312604769 Fax: +27 312604609 Email: BREC@ukzn.ac.za

\section{Competing interests}

The authors declare that they have no competing interests

\section{Consent for publication}

Not applicable

\section{References}

1. Anlaakuu P, Anto F. Anaemia in pregnancy and associated factors: a cross sectional study of antenatal attendants at the Sunyani Municipal Hospital, Ghana. BMC Research Notes. 2017;10(1):402. 
2. Lebso M, Anato A, Loha E. Prevalence of anemia and associated factors among pregnant women in Southern Ethiopia: A community based cross-sectional study. PloS one. 2017;12(12):e0188783.

3. Finkelstein JL, Herman HS, Guetterman HM, Peña-Rosas JP, Mehta S. Daily iron supplementation for prevention or treatment of iron deficiency anaemia in infants, children, and adolescents. The Cochrane Database of Systematic Reviews. 2018;2018(12).

4. Tunkyi K, Moodley J. Prevalence of anaemia in pregnancy in a regional health facility in South Africa. South African Medical Journal = Suid-Afrikaanse Tydskrif Vir Geneeskunde. 2015;106(1):1014.

5. Singh R, Singh AK, Gupta S, Singh HK. Correlates of anemia in pregnant women. Indian journal of community health. 2015;27(3):351-5.

6. Debnath A, Debbarma A, Debbarma SK, Bhattacharjya H. Proportion of anaemia and factors associated with it among the attendees of the antenatal clinic in a teaching institute of northeast India. Journal of family medicine and primary care. 2021;10(1):283-8.

7. Waheeda S.K Kara JC, Magafu M. Mzigaba, Jing Mao and Fabian P. Mghanga. Anaemia in pregnancy in Southern Tanzania: Prevalence and associated risk factors. African Journal of Reproductive Health. 2020;24(3):154-60.

8. Wemakor A. Prevalence and determinants of anaemia in pregnant women receiving antenatal care at a tertiary referral hospital in Northern Ghana. BMC Pregnancy And Childbirth. 2019;19(1):495-.

9. Woldegebriel AG, Gebregziabiher Gebrehiwot G, Aregay Desta A, Fenta Ajemu K, Berhe AA, Woldearegay TW, et al. Determinants of Anemia in Pregnancy: Findings from the Ethiopian Health and Demographic Survey. Anemia. 2020;2020:2902498.

10. Tunkyi K, Moodley J. Anemia and pregnancy outcomes: a longitudinal study. The Journal of Maternal-Fetal \& Neonatal Medicine. 2018;31(19):2594-8.

11. Mishra A, Marwah S, Divedi P, Dewan R, Ahluwalia H. A Cross-Sectional Study of Barriers in Prevention of Anemia in Pregnancy. Cureus. 2021;13(1):e12802.

12. Chowdhury HA, Ahmed KR, Jebunessa F, Akter J, Hossain S, Shahjahan M. Factors associated with maternal anaemia among pregnant women in Dhaka city. BMC women's health. 2015;15:77.

13. Hailu T, Kassa S, Abera B, Mulu W, Genanew A. Determinant factors of anaemia among pregnant women attending antenatal care clinic in Northwest Ethiopia. Tropical diseases, travel medicine and vaccines. 2019;5:13.

14. Ssentongo P, Ba DM, Ssentongo AE, Ericson JE, Wang M, Liao D, et al. Associations of malaria, HIV, and coinfection, with anemia in pregnancy in sub-Saharan Africa: a population-based cross-sectional study. BMC pregnancy and childbirth. 2020;20(1):379.

15. Stephen G, Mgongo M, Hussein Hashim T, Katanga J, Stray-Pedersen B, Msuya SE. Anaemia in Pregnancy: Prevalence, Risk Factors, and Adverse Perinatal Outcomes in Northern Tanzania. Anemia. 2018;2018:1846280-.

16. Ugwu NI, Uneke CJ. Iron deficiency anemia in pregnancy in Nigeria-A systematic review. Nigerian journal of clinical practice. 2020;23(7):889-96. 
17. Omote V, Ukwamedua HA, Bini N, Kashibu E, Ubandoma JR, Ranyang A. Prevalence, Severity, and Correlates of Anaemia in Pregnancy among Antenatal Attendees in Warri, SouthSouthern Nigeria: A Cross-Sectional and Hospital-Based Study. Anemia. 2020;2020:1915231.

18. Gedefaw L, Ayele A, Asres Y, Mossie A. Anaemia and associated factors among pregnant women attending antenatal care clinic in Walayita Sodo town, Southern Ethiopia. Ethiopian journal of health sciences. 2015;25(2):155-64.

19. Mekonnen FA, Ambaw YA, Neri GT. Socio-economic determinants of anemia in pregnancy in North Shoa Zone, Ethiopia. PloS one. 2018;13(8):e0202734.

20. Wiegersma AM, Dalman C, Lee BK, Karlsson H, Gardner RM. Association of prenatal maternal anemia with neurodevelopmental disorders. JAMA psychiatry. 2019;76(12):1294304.

21. Tunkyi K, Moodley J. Anaemia in pregnancy in a setting of high HIV prevalence rates. Southern African Journal of Infectious Diseases. 2017;32(4):138-41.

22. Swaziland MoH. Swaziland Intergrated HIV Management. Mbabane2015.

23. Siteti MC, Namasaka SD, Ariya OP, Injete SD, Wanyonyi WA. Anaemia in pregnancy: Prevalence and possible risk factors in Kakamega County, Kenya. Science journal of public health. 2014;2(3):216-22.

24. Organisation WH. NLiS Country Profile: Lesotho2016. Available from: http://apps.who.int/nutrition/landscape/report.aspx?iso=Iso.

25. Organisation WH. NLiS Country Profile: Botswana2016. Available from: http://apps.who.int/nutrition/landscape/report.aspx?iso=bwa.

26. Okube OT, Mirie W, Odhiambo E, Sabina W, Habtu M. Prevalence and factors associated with anaemia among pregnant women attending antenatal clinic in the second and third trimesters at pumwani maternity hospital, Kenya. Open Journal of Obstetrics and Gynecology. 2016;6(1):16-27.

27. Hameed H, Hameed A, Bashir S, Akram S, Arshad M. Study of Prevalence of Anaemia among Pregnant Women and Its Correlation with Different Risk Factors. Drug Des. 2018;7(158):21690138.1000158.

28. El Ashiry A, El Ghazali S, Habil I. Prevalence and determinants of anaemia in third trimester pregnancy in Fayoum governorate-Egypt. Acta Medica Mediterranea. 2014;30(10):1045-51.

29. Nonterah EA, Adomolga E, Yidana A, Kagura J, Agorinya I, Ayamba EY, et al. Descriptive epidemiology of anaemia among pregnant women initiating antenatal care in rural Northern Ghana. African Journal Of Primary Health Care \& Family Medicine. 2019;11(1):e1e7.

30. Gebre A, Mulugeta A. Prevalence of anemia and associated factors among pregnant women in North Western zone of Tigray, Northern Ethiopia: a cross-sectional study. Journal of nutrition and metabolism. 2015;2015.

31. Lin L, Wei Y, Zhu W, Wang C, Su R, Feng H, et al. Prevalence, risk factors and associated adverse pregnancy outcomes of anaemia in Chinese pregnant women: a multicentre retrospective study. BMC pregnancy and childbirth. 2018;18(1):111. 
32. Madu AJ, Ughasoro MD. Anaemia of chronic disease: an in-depth review. Medical Principles and Practice. 2017;26(1):1-9.

33. Ononge $S$, Campbell O, Mirembe F. Haemoglobin status and predictors of anaemia among pregnant women in Mpigi, Uganda. BMC research notes. 2014;7:712. 